\title{
Heparin-induced thrombocytopenia as a cause of prolonged low platelet count in a patient with thrombotic thrombocytopenic purpura treated with plasmapheresis
}

\begin{abstract}
Agata Winiarska, Norbert Kwella and Tomasz Stompór ${ }^{\bowtie}$ in Olsztyn, Poland

Thrombotic thrombocytopenic purpura (TTP) is a rare disorder belonging to thrombotic microangiopathies (TMA) and is caused by functional deficiency of the ADAMTS-13 metalloproteinase. Plasma exchange (PE) remains the treatment of choice in this disease. Here, were describe the case of a patient who apparently recovered from TTP following multiple sessions of PE, but remained thrombocytopenic. Careful analysis revealed the development of heparin-induced thrombocytopenia (HIT) that precluded platelet count (PLT) normalization. Full normalization of PLT followed discontinuation of PE and low-molecular weight heparin.
\end{abstract}

Department of Nephrology, Hypertension and Internal Medicine, Chair of Internal Medicine, Medical Faculty, University of Warmia and Mazury

Key words: heparin-induced thrombocytopenia, plasma exchange, thrombotic thrombocytopenic purpura

Received: 17 September, 2016; revised: 20 October, 2016; accepted: 20 October, 2016; available on-line: 05 April, 2017

e-mail: stompin@mp.pl

Abbreviations: HIT, heparin-induced thrombocytopenia; PE, plasma exchange; PLT, precluded platelet count; TMA, Thrombotic thrombocytopenic purpura; TTP, thrombotic thrombocytopenic purpura

\section{INTRODUCTION}

Thrombotic thrombocytopenic purpura (TTP) is a rare disorder belonging to thrombotic microangiopathies (TMA) and is caused by functional deficiency of the ADAMTS-13 metalloproteinase (under physiolog- ical conditions, ADAMTS-13 cleaves the von Willebrand factor multimers). Clinical presentation includes: consciousness disorders, low platelet (PLT) count, microangiopathic hemolityc anemia (MAHA), clinical and laboratory signs of hemolysis (i.e. low haptoglobin level, high lactic dehydrogenase (LDH) activity). Deficiency of ADAMTS-13 activity can be hereditary (due to genetic defects) or acquired, secondary to anti-ADAMTS-13 antibody formation (the latter occurring with the frequency of 3-6/1000000 population, mainly affecting young women) (Tersteeg et al., 2016). Heparin-induced thrombocytopenia (HIT) is an adverse drug reaction occurring after exposure to heparin, usually between 5-10 days after first administration, caused by heparin dependent, platelet-activating $\mathrm{IgG}$ antibodies. Certain signs and symptoms of these two clinical entities may overlap and thus need careful differential diagnosis (Warkentin, 2015).

\section{CASE REPORT}

A 58-year-old patient was referred to our department with suspicion of the haemolytic-uremic syndrome. On admission, the patient's general status was severe, with impaired consciousness, mild impairment of renal function and signs of thrombocytopenic purpura (previous medical history not relevant). Lab test results were as follows: $\mathrm{Hb} 7.7 \mathrm{~g} / \mathrm{dL}, \mathrm{PLT} 16 \times 10^{3} / \mu \mathrm{l}$, LDH 1681 $\mathrm{IU} / \mathrm{L}$, haptoglobin $1 \mathrm{mg} / \mathrm{dL}$, serum urea $87 \mathrm{mg} / \mathrm{dL}$,

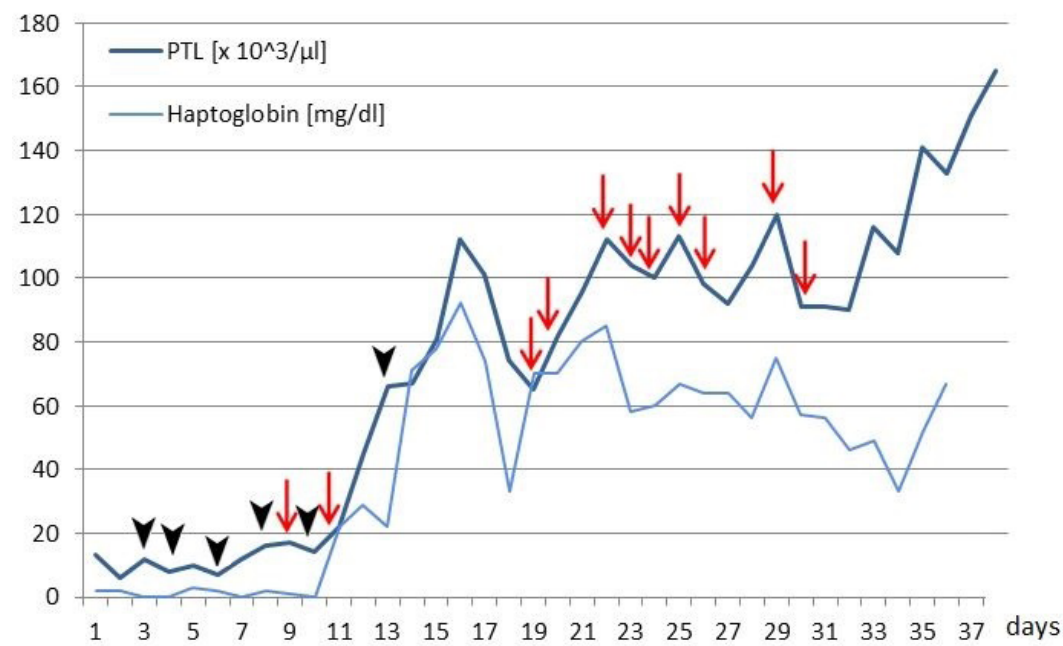

Figure 1. Platelet count and serum hemoglobin throughout the treatment period. Arrows, PE with LMWH; Arrowheads, PE without LMWH. 
serum creatinine $1.7 \mathrm{mg} / \mathrm{dL}$, eGFR-MDRD $41.9 \mathrm{~mL} /$ $\min . / 1.73 \mathrm{~m}^{2}$. Based on the above clinical laboratory manifestations, daily PE procedures were started immediately. Diagnosis was confirmed by very low plasma activity of ADAMTS-13 $(<0.1 \%)$, with presence of anti-ADAMTS-13 antibodies. During the course of hospitalization, $17 \mathrm{PE}$ procedures were performed, with replacement of 3600 to $5632 \mathrm{~mL}$ of fresh frozen plasma (FFP) during each procedure (initially - without anticoagulation due to low PLT, and later on - using low molecular weight heparin [LMWH, enoxaparin]). Treatment with high-dose corticosteroids was also initiated. After 22 days of treatment (and 11 PE procedures) PLT count stabilized in the range between $90 \times 10^{3}$ and $100 \times 10^{3} /$ $\mu \mathrm{L}$, but no further PLT count improvement was observed despite PE continuation and full normalization of LDH activity and haptoglobin concentration (Fig. 1). This discrepancy raised the suspicion of heparin-induced thrombocytopenia (HIT). Paradoxically, PE withdrawal resulted in full normalization of the PLT count. Blood test drawn immediately after the suspicion of HIT was raised revealed the presence of antibodies against heparin/platelet factor 4 complex.

\section{DISCUSSION}

Literature describing development of HIT in the context of TMA is scarce. We were able to find only two case reports, describing four cases in total, and one cross-sectional study performed in patients who underwent stem cell transplantation. Studt et al. reported three patients referred due to TMA (haemolytic-uremic syndrome [HUS] secondary to disseminated prostate cancer, typical HUS due to infection of Shiga-toxin producing E. coli, and TTP with confirmed severe ADAMTS-13 deficiency, respectively). Two of them were treated with hemodialysis and PE, whereas the third one - with PE only (in all cases - using unfractionated heparin for anticoagulation). As in our case - persistence or recurrence of thrombocytopenia despite improvement in other clinical and laboratory parameters raised the suspicion of HIT; in all cases, HIT was further confirmed with anti-heparin/PF4 antibody testing (Studt et al., 2013). Benke and Moltzan described a vice versa scenario, i.e. TTP that followed HIT. The patient first developed HIT while on treatment with unfractionated heparin following CABG surgery, and later on displayed features of hemolysis, worsening of thrombocytopenia, moderate-degree renal failure and certain neurological symptoms (concordant with diagnosis of TTP). This patient also successfully recovered from TTP following eight sessions of heparin-free PE with FFP and steroids (Benke \& Moltzan, 2005). Finally, Takatsuka and coworkers described the group of stem cell transplant recipients (SCT; performed for different hematogenous malignancies) who developed $(\mathrm{N}=10)$ or did not develop $(\mathrm{N}=39)$ TMA. The serum level of PF-4 was assessed in all patients before and after transplantation (all were treated with heparin to prevent a veno-occlusive disease). The authors had found that patients who subsequently developed TMA were characterized with a significantly higher post-transplant PF4 level vs those who remained TMA-free (baseline levels were not different). The authors had hypothesized that an increased PF-4 level may predispose to the development of TMA (Takatsuka et al., 2005). However, it seems that this interesting finding did not attract attention of transplant haematologists since no further studies were performed on this topic.

In summary, to the best of our knowledge, we report on the second case ever found in the literature of a patient who developed HIT following acquired T'TP, initially treated with success using $\mathrm{PE}$ and heparin for TTP, and later on recovering from HIT after withdrawal of PE and heparin.

\section{REFERENCES}

Tersteeg C, Verhenne S, Roose E, Schelpe AS, Deckmyn H, De Meyer SF, Vanhoorelbeke K. (2016) ADAMTS13 and anti-ADAMTS13 autoantibodies in thrombotic thrombocytopenic purpura - current perspectives and new treatment strategies. Expert Rev Hematol 9: 209-221. doi: 10.1586/17474086.2016.1122515

Warkentin TE (2015) Heparin-induced thrombocytopenia. Curr Opin Crit Care 21: 576-585. doi: 10.1097/MOH.0000000000000273.

Studt J-D, Binet I, Nair G, Schanz U (2013) Heparin - induced thrombocytopenia associated with thrombotic microangiopathy. Hamostaseologie 33: 160-163. doi: 10.5482/HAMO-13-03-0012

Benke S, Moltzan C (2005) Co-existence of heparin-induced thrombocytopenia and thrombotic thrombocytopenic purpura in a postoperative cardiac surgery patient. Am I Hematol 80: 288-291

Takatsuka H, Nakajima T, Nomura K, Wakae T, Toda A, Itoi H, Okada M, Misawa M, Hara H (2005). Heparin-induced thrombocytopenia antibody and the pathogenesis of thrombotic microangiopathy after stem cell transplantation. Clin Transplant 19: 418-422 